THE ASTROPHYSICAL JoURNAL, 469:542-553, 1996 October 1

(C) 1996. The American Astronomical Society. All rights reserved. Printed in U.S.A.

\title{
EFFECTS OF SHOCKS ON EMISSION FROM CENTRAL ENGINES OF ACTIVE GALACTIC NUCLEI. I.
}

\author{
R. Sivron, ${ }^{1}$ D. CADITZ, AND S. Tsuruta \\ Physics Department, Montana State University, Bozeman, MT 59717 \\ Received 1994 August 15 ; accepted 1996 April 23
}

\begin{abstract}
In this paper we show that perturbations of the accretion flow within the central engines of some active galactic nuclei (AGNs) are likely to form shock waves in the accreting plasma. Such shocks, which may be either collisional or collisionless, can contribute to the observed high-energy temporal and spectral variability. Our rationale is the following: Observations show that the continuum emission probably originates in an optically thin, hot plasma in the AGN central engine. The flux and spectrum from this hot plasma varies significantly over light crossing timescales. Several authors have suggested that macroscopic perturbations contained within this plasma are the sources of this variability. In order to produce the observed emission the perturbations must be radiatively coupled with the optically thin hot matter and must also move with high velocities. We suggest that shocks, which can be very effective in randomizing the bulk motion of the perturbations, are responsible for this coupling. Shocks should form in the central engine, because the temperatures and magnetic fields are probably reduced below their virial values by radiative dissipation. Perturbations moving at Keplerian speeds, or strong nonlinear excitations, result in supersonic and super-Alfvénic velocities leading to shock waves within the hot plasma.

We show that even a perturbation smaller than the emitting region can form a shock that significantly modifies the continuum emission in an AGN, and that the spectral and temporal variability from such a shock generally resembles those of radio-quiet AGNs. As an example, the shock inducing perturbation in our model is a small main-sequence star, the capturing and eventual accretion of which are known to be a plausible process. We argue that shocks in the central engine may also provide a natural triggering mechanism for the "cold" component of Guilbert \& Rees two-phase medium and an efficient mechanism for angular momentum transfer. Current and future missions, such as $A S C A, X T E, X M M, A X A F$, and $A S T R O-E$ may determine the importance of shock-related emission from the central engines of AGNs.
\end{abstract}

Subject headings: galaxies: nuclei - galaxies: Seyfert - quasars: general — shock waves

\section{INTRODUCTION}

Recent observations indicate that the continuum emission from AGNs originates within a hot plasma (e.g. Mushotzky, Done, \& Pounds 1993, hereafter MDP93, and references therein). Variability arguments suggest that the plasma is contained within a region of size $R_{\mathrm{X}} \sim t_{d} c$, where $t_{d}$ is the flux doubling timescale. Within the commonly accepted accretion model for AGNs, the hot plasma is probably located in the vicinity of a central supermassive black hole (Rees 1984, and references therein; Blandford 1990, hereafter B90). In this model, most of the continuum emission originates from within about twenty gravitational radii of the black hole in the region generally referred to as the AGN central engine.

An extensively considered accretion model of AGNs, the simple optically thick geometrically thin disk model that does not include hot plasma, does not adequately explain the continuum UV-to- $\gamma$ ray spectrum from AGNs. Several modifications to the thin disk geometry have been proposed: (1) the geometrically thick accretion disk supported by radiation pressure (Abramowicz, Calvani, \& Nobili 1980) with a geometry that is susceptible to global perturbations (Papaloizou \& Pringle 1984), (2) the geometrically thick, optically thin disk model with two-temperature rela-

1 sivron@physics.montana.edu. tivistic plasmas, first suggested for an $\mathrm{X}$-ray binary (Shapiro, Lightman, \& Eardley 1976), but which has been extended to AGN counterparts (e.g., Rees et al. 1982; White \& Lightman 1989, hereafter WL89; Tritz \& Tsuruta 1989; Narayan \& Yi 1994), and (3) a disk-corona model (Liang \& Price 1977; Haardt \& Maraschi 1991; Życki, CollinSouffrin, \& Czerny 1995, hereafter ZCC95; Tsuruta \& Kellen 1995, hereafter TK95). For the two-temperature torus model Narayan \& Yi (1994) propose that at lower accretion rates advection lowers the efficiency of the accretion process and stabilizes the otherwise unstable hot electrons, which suggests that this model may be more appropriate for sub-Eddington sources (see also Artemova et al. 1996). A common feature of all models that attempt to reconstruct the higher energy $(\sim 1 \mathrm{keV}-1 \mathrm{MeV})$ power-law spectrum is the introduction of an optically thin hot plasma (with the electron temperature $T_{e}>10^{8} \mathrm{~K}$ ) that may dominate the emission from the central engine.

Large-amplitude temporal variability of the hot plasma emission is present in at least $50 \%$ of radio-quiet $\mathrm{AGNs}$, and significant spectral variability of similar timescales is also very common (MDP93, and references therein; Green 1993; McHardy 1988). The temporal and spectral variability has been generally explained in the context of perturbations on the thin, cold disk model, but rarely in the context of the geometrical models mentioned above. Models for the variability of radio-quiet AGNs include, for 
$=$

.

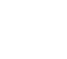


example, the hot spots model (Abramowicz 1990; Wiita 1993, hereafter W93), where a multitude of randomly distributed flares or "hot spots" embedded within a thin disk are responsible for the total variability. Other models also assume randomly distributed perturbations on the thin disk (Pudritz \& Fahlman 1982; Devries \& Kuijpers 1989). The temperature of the thin disk in the central engine is found to be of order $T \sim 10^{6} \mathrm{~K}$ giving a sound speed of order $c_{s} \sim$ $10^{-2} c$ or less (Frank, King, \& Raine 1992, hereafter FKR92). The total volume affected by a single perturbation is therefore too small to easily account for the doubling of the entire continuum luminosity, making these models dependent on the existence of many perturbations of characteristic size, time, and brightness occurring on the disk surface. In contrast, within the hot plasma region, a disturbance may propagate at a much greater speeds, affecting a larger volume of the continuum emitting material. A single perturbation effectively coupled to the hot plasma may transfer enough energy to the radiating region within typical flux doubling timescales to account for the observed continuum variability.

Shocks have been investigated in the context of radioloud AGNs with shocks propagating down a jet and interacting with irregularities in the jet material (Qian et al. 1991), or other forms of shocks (W93). In this paper we investigate the possibility that a strong perturbation, moving at supersonic or super-Alfvénic speeds in the hot plasma of the AGN central engine, forms a shock that modifies the continuum emission (see also Sivron \& Tsuruta 1994). Such shocks may effectively couple macroscopic perturbations with the optically thin hot matter, modifying the continuum spectrum. Shocks are also effective in randomizing the bulk motion of the perturbations, which may have important consequences for the outward transfer of angular momentum.

In our work, we utilize recent studies of the effect of pairs on the central engine structure (Guilbert \& Rees 1988, hereafter GR88; Lightman \& White 1988, hereafter LW88; Coppi \& Blandford 1990, hereafter CB90; Sivron \& Tsuruta 1993, hereafter ST93; and Ghisellini \& Haardt 1994, hereafter GH94). These authors suggest that in compact central engines, dampening processes readily cool a significant fraction of the hot matter, radiating the excess energy away. The cooled matter then forms a cold "phase" component. We show that in central engines with nearEddington luminosity the postshock matter is, in effect, an "increased compactness" region in which the shocked matter is cooled. The shocks are thus a natural mechanism by which radiative power is increased and cold phase matter is created. The cooling of postshock matter results in radiation from the shock front, which may be responsible for the "soft" flares, whereas cold phase matter behind the shock front may be responsible for the relatively hard "dips" in the light curve (see \$ 4).

For mathematical simplicity we use a model in which the source of the perturbations is local, for example, a small main-sequence star. Such a star can be captured and eventually accreted into the central engine (Syer, Clarke, \& Rees 1990, hereafter SCR90). For a wide range of accretion parameters, such an event naturally results in the creation of shocks. Such shocks can significantly modify the light curve from the $\mathrm{X}$-ray emitting region of AGNs.

In $\S 2$ we show that macroscopic inhomogeneities, which we assume travel at Keplerian speeds, naturally posess supersonic or super-Alfvénic speeds when present in the hot plasma of the AGN central engine for the three models discussed above. In $\S 3$ we show that the shocks effectively couple the perturbations with the hot plasma, and large enough perturbations can therefore result in the modification of the total emission. In $\S 4$ we calculate the effects of a large shock on the light curve of a model AGN. The main purpose of this exercise is to demonstrate the type of spectral variability expected when shocks modify the emission from the central continuum source. We discuss our results in $\S 5$, and present our conclusions in $\S 6$.

\section{SHOCK FORMATION}

Recent observations as well as theoretical analyses suggest that hot plasma must exist in the central engine. From an observational point of view, the variable continuum of nearly power-law shape in the X-ray to $\gamma$-ray bands could be explained as emission from hot plasma radiating synchrotron self-Compton or Comptonized bremsstrahlung radiation from an accretion disk, filaments, or clouds (B90; ST93; GH94; TK95). Theory predicts that the simple thin accretion disk will be disrupted within a critical radius where heating due to gravitational and viscous energy release surpasses the cooling by bremsstrahlung, the temperature rises and the gas pressure disrupts the thin disk (Rees et al. 1982; B90). Other plasma instabilities may also disrupt the thin disk (see, e.g., W93). The accretion flow inside this radius is not a simple thin disk and it may form a geometrically thick torus (cases [1] and [2] above), or a disk corona structure with dominant radiation coming from corona (Haardt \& Maraschi 1991).

In order to describe the physical conditions within the central engine in the above models we use the following parameters: The dimensionless radial distance to the supermassive black hole is $r=R / R_{g}$, where $R_{g}=G M / c^{2}$ is the gravitational radius. The bulk of X-ray continuum is thought to originate from within $R_{\mathrm{X}} \sim 20 R_{g}$. The central black hole mass, $M$, for Seyfert galaxies and radio-quiet quasars has been estimated from X-ray time variability data to be $M>\sim 10^{6} M_{\odot}$, and we therefore write the black hole mass in terms of $M_{6}=M / 10^{6} M_{\odot}$. The accretion rate, $\dot{M}$, has been related to the X-ray luminosity, $L_{\mathrm{X}}$, through $\dot{M}=$ $L_{\mathrm{X}} /\left(\eta c^{2}\right)$, where $\eta$ is the efficiency of converting gravitational energy of the accreted matter into radiation. Luminosities of order $L_{\mathrm{X}} \sim 10^{41-44} \mathrm{erg} \mathrm{s}^{-1}$ are typical for the $2-10 \mathrm{keV}$ band. The dimensionless accretion rate is $\dot{m}=\dot{M} / \dot{M}_{\mathrm{E}}$, where

$$
\dot{M}_{\mathrm{E}}=\frac{4 \pi G M m_{p}}{\left(\sigma_{\mathrm{T}} c\right)},
$$

is the Eddington accretion rate, $m_{p}$ is the proton mass, and $\sigma_{\mathrm{T}}$ is the Thompson cross section. Assuming $\eta \sim 0.1$ and $M_{6} \sim 1$ we find typical accretion rates of order $0.01<$ $\sim \dot{m}<\sim 10$.

\subsection{Collisional Shocks}

That collisional shocks form and Rankine-Hugoniot relations can be written, when wave steepening occurs in relativistic thermal plasmas was shown by Taub (1948). Collision-dominated waves and shocks may be present when the particle deflection length is smaller than the size of 
a typical large perturbation in the central engine, i.e., when

$$
\lambda \sim \frac{2\left(k T_{e}\right)^{2}}{\pi n_{i} e^{4} \ln \Lambda}<R_{g},
$$

where $n_{i}$ is the ion number density, $T_{e}$ is the electron temperature, and the Coulomb logarithm is here taken to be $\ln \Lambda \sim 20$ (see, e.g., FKR92). Assuming quasi-spherical accretion geometry with solid angle $\Omega$, we may easily relate the disk density to the accretion rate:

$$
\dot{M}=\Omega R^{2} \mu m_{p} n_{i} v_{\text {acc }} .
$$

Here $\mu m_{p}$ is the average ion mass and $v_{\text {acc }}$ is the radial accretion velocity. Equations (2) and (3) then give a lower limit to the accretion rate for AGNs that may support collisional shocks:

$$
\dot{m} \geq 0.08\left(\frac{\Theta_{e}}{\Theta_{v}}\right)^{2}\left(\frac{r}{20}\right)^{-1 / 2}\left(\frac{\Omega}{4 \pi}\right)\left(\frac{v_{\mathrm{acc}}}{v_{\mathrm{ff}}}\right),
$$

where $\Theta_{e}=k T_{e} / m_{e} c^{2}$ is the dimensionless electron temperature and $\Theta_{v}=1 / r$ is the dimensionless virial temperature. One can see that collision-dominated waves may form in the near or super-Eddington regime. This result is hardly surprising, since it is related to that of GR88, which claims that the central engine is almost always optically thick for near Eddington luminosity, with the main difference being that the mean free path for photons in GR88 is replaced by the deflection length for electron collisions. We can compare the above result with the condition in Rees et al. $(1982), \dot{m}_{\mathrm{cr}} \leq 50 \alpha^{2}$ on the maximal accretion rate such that ions and electrons decouple to form a two-temperature ion torus. Here $\alpha$ is similar to the parameter in the standard $\alpha$ accretion model, which relates the stress and pressure in a thin disk. We see that even two-temperature tori can marginally support collisional waves.

For the cases in which the shocks are collision dominated we use the thermodynamic parameters, such as the adiabatic constant $\Gamma$, the speed of sound $c_{s}$, and others, from a paper on shocks in relativistic collisional plasmas (Iwamoto 1989 , hereafter I89, and references therein). The speed of sound in the plasma is

$$
\begin{aligned}
\left(\frac{c_{s}}{c}\right)^{2}=\left(1+\frac{1}{1+\zeta^{2}\left\{K_{2}^{\prime \prime}(\zeta) / K_{2}(\zeta)\right.}-[\right. & {\left.\left[K_{2}^{\prime}(\zeta) / K_{2}(\zeta)\right]^{2}\right\} } \\
\times & {\left[2-\zeta \frac{K_{2}^{\prime}(\zeta)}{K_{2}(\zeta)}\right]^{-1}, }
\end{aligned}
$$

where $\zeta=m c^{2} / k T$, and $K_{2}(\xi)$ is the modified Bessel function of the second kind, which naturally arises from the inclusion of relativistic energies in the exponent of the partition function (I89, eq. [A22]).

The equilibrium temperature does not quite reach its virial value because the outward transfer of angular momentum, which also gives rise to radiation, is dissipative. We therefore write the dimensionless ion temperature, $\Theta_{i}=k T_{i} / \mu m_{p} c^{2}$, as a fraction $\delta$ of the virial temperature,

$$
\Theta_{i}=\delta \Theta_{v}
$$

where $\delta \sim<1$.

The ideal gas law, which holds for collisional plasmas at high temperatures (I89, eq. [A8]), is a reasonable approximation for the equation of state, and in the ion-dominated case the speed of sound in equation (5) reduces to

$$
c_{s} \sim \sqrt{\Gamma \delta} r^{-1 / 2} c,
$$

where we assumed $\Theta_{i}<1$. If the electrons in the preshock region are coupled with the ions, the electron speed of sound is calculated using the exact form of equation (5). This yields a speed that is roughly $c_{s e} \sim c / 3$, because the electrons are relativistic and may be described by a simple equation of state as $p_{e} \sim \epsilon_{e} / 3$, where $p_{e}$ and $\epsilon_{e}$ are the pressure and the energy, respectively. Cases in which the preshock electrons are decoupled from the ions are not described in I89 or other references, and are therefore not discussed in this paper. We note, however, that the limit in equation (10) at the end of this section is still valid.

An object or perturbation in the accreting plasma that is more compact than the surrounding material, so that it cannot thermalize or lose its angular momentum on dynamical timescales, moves at a velocity comparable to its Keplerian or free-fall velocity (see also $\$ 3.1$ and the next paper in this series, Sivron, Tsuruta, \& Caditz 1996b, hereafter STC96). The relative speed of such a compact object is therefore

$$
v_{*}=\left|v_{*}-v_{\theta}\right|=b r^{-1 / 2} c,
$$

where $v_{*}$ is the velocity of the object, $v_{g}$ is the velocity of the accreting matter and $b$ is a geometrical factor of order unity. The Mach number is therefore given by

$$
\mathscr{M}=\frac{v_{*}}{c_{s}} \sim \delta^{-1 / 2} .
$$

This Mach number is insensitive to the object's distance from the black hole because both the speed of sound and the typical velocity are approximately proportional to the square root of the virial energy. For a wide range of accretion rates $0.003<\delta<1$ (I89; WL89), which results in

$$
0.8<\mathscr{M}<16 \text {, }
$$

where for the upper limit we substitute $\Gamma=4 / 3$. This result suggests that shock waves are likely to form in AGN central engines that satisfy the constraint imposed by equation (4). The Mach surface opening angle, $\phi \sim 2 \arcsin \left(c_{s} / v_{*}\right)$, may be significant (see $\S 4$ ). The shocked surface formed behind a small perturbation roughly takes a conical shape that is likely modified by the presence of strong gravitational and shear forces, radiation pressure, turbulent flows, and density gradients within the central engine.

\subsection{Collisionless Shocks}

For accretion rates lower than the limit of equation (4) the deflection length is larger than the characteristic size of the central engine, and collision-dominated shocks do not form. However, magnetic and electric fields and turbulence are expected to be present and collisionless shocks may occur (Kennel, Edmiston, \& Hada 1985, hereafter KEH85). In this case, the plasma state across the shock is determined not by the binary collisions, but by collective interactions between particles and self-consistently generated electric and magnetic fields. Plasma heating due to these shocks is the result of various microinstabilities that perturb the fields and particle distribution (Papadopoulos 1985, hereafter P85; Winske 1985).

Collisionless shocks may be present when the Larmor radius is smaller than the central engine. The thickness of collisionless shocks is expected to be from several Larmor radii for quasi-perpendicular shocks to several hundred times the Larmor radius for quasi-parallel shocks (FKR92). 
Note, however, that this result has not yet been confirmed for the near-relativistic plasma treated in this paper. For hydrogen-dominated plasmas the ion Larmor radius is roughly

$$
\begin{aligned}
& R_{L i} \sim(0.03 \mathrm{~cm}) \mathscr{B}^{-1} \dot{m}^{-1 / 2} M_{6}^{1 / 2} r^{3 / 4} \\
& \times\left(\frac{\Theta_{i}}{\Theta_{v}}\right)^{1 / 2}\left(\frac{\Omega}{4 \pi}\right)^{1 / 2}\left(\frac{v_{\mathrm{acc}}}{v_{\mathrm{ff}}}\right)^{1 / 2},
\end{aligned}
$$

where $\mathscr{B}=B / B_{v}$ is the magnetic field in units of the virial magnetic field, which can be found from the relation $B_{v}^{2} / 8 \pi=G M \rho_{g} / R$, and we have used equations (1) and (3). AGN central engines may support magnetic fields of up to the virial value of $\sim 10^{4} \mathrm{G}$ at $r \sim 20$ (B90). The $\sim 10^{-8} \mathrm{G}$ fields needed for the Larmor radius to be smaller than a perturbation in the central engine are easily obtained from matter accreted from stars. This limit is almost always satisfied for the fields anticipated in the central engine (see, e.g., B90).

For collisionless shocks in the central engines of AGNs one needs to use the kinetic equations for relativistic plasmas. Unfortunately, such equations are not fully developed yet (Eilek \& Hughes 1991), but several criteria from the studies of relativistic shocks can be used. For example, Barnes (1985) found that Alfvénic nonlinear waves steepen and become shocks. We therefore speculate that superAlfvénic motions result in collisionless shocks.

The Alfvénic velocity is

$$
c_{\mathrm{A}}=\frac{B}{\sqrt{4 \pi n_{i} m_{p}}} \sim c \mathscr{B} r^{-1 / 2},
$$

which is comparable to $c_{s}$ for virial fields. The superAlfvénic Mach number at that distance for Keplerian speed perturbations is thus

$$
\mathscr{M}_{\mathrm{A}} \sim \mathscr{B}^{-1}=B_{v} / B .
$$

It is therefore expected that strong collisionless shocks may form in the central engines for moderate magnetic fields, and moderate shocks may form for near virial fields. Instabilities that disrupt the thin disk may also result in the creation of a disk corona structure in which the corona is responsible for emitting the continuum for low enough accretion rates (Haardt \& Maraschi 1991; Svensson \& Zdziarski 1994; ZCC95). In the corona of a disk corona system the corona accretion rate is much smaller than in the tori models. In order to find the appropriate density for such corona one needs a model in which both disk and corona accretion are treated consistently. Such treatment was recently taken by ZCC95. Using Figure 5 in that paper for accretion rates of $\dot{m} \sim 0.001$ to $\dot{m} \sim 0.1$ and using equation (12) one finds

$$
c_{\mathrm{A}} \geq\left(3 \times 10^{6-7} \mathrm{~cm} \mathrm{~s}^{-1} \mathrm{G}^{-1}\right) B,
$$

and thus at $r \sim 20$

$$
\mathscr{M}_{\mathrm{A}} \sim\left(3 \times 10^{3-4}\right) \mathscr{B}^{-1} .
$$

In both equations (13) and (15) the Alfvenic Mach number generally exceeds unity because the magnetic fields cannot quite reach their virial value. This is because energy from the magnetic field may be utilized for the outward transfer of angular momentum and/or for dissipation, which in collisionless plasmas may include, for example, reconnection events in the postshock region.

\section{FORMATION AND PROPERTIES OF THE SHOCKED MATTER}

\subsection{The Shock Creating Perturbations}

Shock creating inhomogeneities in AGN central engines may be the result of nonlinear growth of global perturbation in thick disks that tend to form planetesimals (Papaloizou \& Pringle 1984; Narayan 1990, and references therein), externally confined plasma sheets and filaments that cannot thermalize on orbital timescales (GR88; ST93; Celotti, Fabian, \& Rees 1992, hereafter CFR92) or captured stars. The simplest physical shock creating perturbation considered in this paper is a main-sequence star. A more general treatment will be presented in our subsequent work (STC96; see also Caditz, Sivron, \& Tsuruta 1996, hereafter CST96). It has been shown that an unbound star can be captured into a tightly bound orbit around a black hole through repeated interactions with a thin disk around the hole (SCR90), and that the probability of such an event in AGNs is even higher for stars in bound orbits (Pineault \& Landry 1994). Once captured the orbit will be circularized and eventually the will be "grounded down" into the disk plane through repeated interactions with the disk. The timescale for circularization can be shorter than that for planarization, which may happen very close to the central source. Therefore, in our model, the orbit of the captured star has been circularized, whereas the orbital plane is still inclined to the disk plane. This situation is expected for a wide range of the original relative inclination angles of these planes.

The minimum conditions on perturbations with the potential to create shocks can be determined from the requirement that they accelerate to supersonic velocities. This condition is met when the gravitational force is greater than the drag force at $\mathscr{M}=1$ :

$$
\frac{G M m_{*}}{R^{2}} \geq \pi D_{*}^{2} p_{*},
$$

where $p_{*} \sim \rho_{g} \mathscr{M}^{2} c_{s}^{2}$ is the pressure on the inhomogeneity surface (see $\S 3.2$ ) and $D_{*}$ is the characteristic size of the inhomogeneity. This condition can be also written in the form

$$
\frac{\rho_{*}}{\rho_{g}} \geq \frac{R_{g} r}{D_{*}},
$$

where $\rho_{*}$ is the average density of the inhomogeneity. This condition is easily met for a typical main-sequence star, which is used as an example shock producing inhomogeneity below.

Equation (17) may be even less restrictive if we include acceleration by radiation pressure and magnetic stresses. The amplitude and size of perturbations needed for shocks to form is therefore rather small, and shocks may form in the wake of large-amplitude nonaxisymmetrical waves in the disk. In this paper the possibility of nonlinear (soliton) shocks is not further developed because the dynamic evolution of the perturbations is strongly dependent on dissipation (see STC96).

\subsection{Power Transferred to the Shock Front} least

The power transferred from the star to the shock is at

$$
P_{\mathrm{sts}} \sim\left(A_{*} p_{*}\right) v_{*},
$$


where $A_{*}=\pi D_{*}^{2}$ is the cross section area of the star and $p_{*}$ is the pressure near the stellar surface, behind the shock.

The pressure at the leading edge of the stellar surface can be estimated from the treatment of shock formation in supersonic nonrelativistic flows past solids of revolution (Landau \& Lifshitz 1987, hereafter LL87):

$$
p_{*} \geq 1.5 \rho v_{*}^{2} \frac{1}{\left[1-1 /\left(5 \mathscr{A}^{2}\right)\right]^{1.5}} \text {. }
$$

The adiabatic constant was chosen to be its highest value of $\Gamma=5 / 3$, which results in a lower limit on $p_{*}$. The power transferred from the star to the shock is thus at least

$P_{\text {sts }} \sim\left(1.5 \times 10^{43} \mathrm{ergs} \mathrm{s}^{-1}\right) \frac{\dot{m} M_{6}}{r^{3}}\left(\frac{4 \pi}{\Omega}\right)\left(\frac{v_{\mathrm{ff}}}{v_{\mathrm{acc}}}\right)\left(\frac{D_{*}}{R_{g}}\right)^{2}$.

The radiation thus emitted can reach the observed luminosity with a single large perturbation, if the shocked material radiates efficiently. For collisionless shocks the ambient pressure is modified to be

$$
p_{\mathrm{A}}=p_{*}+\frac{B^{2}}{8 \pi}
$$

and the pressure from equation (19) is somewhat larger, especially with $\Gamma \sim 4 / 3$ for the heat capacity ratio when the magnetic effect is dominant.

We next argue that if the accretion is already near its Eddington limit shocks will provide the mechanism for making the postshock region radiative. We separately treat the collisional and collisionless shock cases.

\subsection{Radiative Efficiency of Shocked Material 3.3.1. Collisional Shocks}

In the near-Eddington regime radiative processes in the postshock region may be extremely efficient, owing to, for example, bremsstrahlung from thermal pairs. We speculate that most of the energy is radiated by pairs that are created in the postshock region. The presence of some pairs is expected in collisional hydrogen plasmas with bulk motion with velocity of order

$$
U \geq\left[\frac{2 m_{e} c^{2}}{m_{p}}\right]^{1 / 2}
$$

(see I89) and in the central engine the bulk motion is of order $v_{*}$, which is generally much larger than $U$ in equation (22).

A more significant pair creation is present in the postshock region because the temperature of the postshock electrons is $\Theta_{e}^{\prime} \gg 1$, and because these electrons are thermalized. We hereafter denote with a prime the postshock quantities. In this scenario the optical depth for pair annihilation is larger than unity, and a high-density pair equilibrium is established in the postshock region. These circumstances were investigated by Done \& Fabian (1989, hereafter DF89), CB90, and others. In their simulations these authors found that the spectrum, which is a power law due to, for example, Comptonization of bremsstrahlung radiation, is flattened because of the increased compactness of the source.

This situation is also likely to apply locally to the postshock region because the optical depth for pair annihilation is of order $\tau_{\mathrm{pa}} \sim 0.4 n_{1}^{\prime} \sigma_{\mathrm{T}} \lambda=5 \Theta_{\mathrm{e}}^{2}$ and $\Theta_{e} \geq 1$. The timescale for mildly relativistic $\left(\Theta_{e} \sim 1\right)$ electrons to thermalize $t_{e} \sim$ $1 /\left(c \sigma_{\mathrm{T}} n_{i}^{\prime} \ln \Lambda\right)$ is shorter than the shock crossing timescale $t_{\mathrm{sw}} \sim \lambda / c_{s}$, and, using equation (2), we find the following ratio to be smaller than unity:

$$
\left[\frac{t_{e}}{t_{\mathrm{sw}}}\right]=\frac{3}{64} \frac{c_{s}}{c} \frac{1}{\Theta_{e}^{2}} \leq 1 .
$$

Here we have used equation (5) and the condition

$$
\frac{n_{i}^{s}}{n_{i}} \geq 4
$$

from I89. The condition of equation (23) is generally fulfilled, and the postshock electrons are therefore thermalized (see also CST96 and STC96, for a more detailed approach).

For the thermalized postshock pair equilibrium described above, the total number density of pairs, $n_{l}^{\prime}$, may be estimated by evaluating the postshock energy of the ions and assuming equipartition. The ion energy is approximately

$$
\frac{\epsilon_{i}^{\prime}}{m_{e} c^{2}} \geq 4 n_{i}\left(1+\frac{m_{p}}{m_{e}} \frac{3 \Theta_{i}^{\prime}}{2}\right)
$$

where we have used the postshock energy from I89 and equation (24).

At $r \sim 20$ the bulk velocity is roughly $v_{*} \sim c / 20^{1 / 2}$, and the postshock temperature is roughly $\Theta_{i}^{\prime}=30 m_{e} / m_{p}$, which translates to more than $\sim 40$ electrons and positrons per postshock ion in equation (25), and we have used equations (8) and (22). Our assumption that at the above location the postshock creation of pairs is unstable for $\dot{m} \sim 0.1$ is supported by Bjornsson \& Svennson 1991 (see also Fig. 1).

Because the density of pairs in the postshock region is so much larger than that of ions in the preshock region, and because bremsstrahlung radiative emmissivity is proportional to the square of the density, the postshock pairs may lose most of their energy to bremsstrahlung radiation. The total bremsstrahlung power per unit volume is of order

$$
j \geq\left(1.2 \times 10^{-23}\right) \mathrm{erg} \mathrm{cm} \mathrm{cm}^{-3} \mathrm{~s}^{-1}\left(n_{i}^{\prime}\right)^{2}\left(\Theta_{e}^{\prime}\right)^{1 / 2},
$$

(e.g., FKR92). The volume of emitting postshock matter is of order $\lambda \pi D_{*}^{2}$, and the power radiated assuming an optically thin source is

$$
P_{b} \sim j \lambda \pi D_{*}^{2}
$$

which, using equations (20), (25), and (26), generally exceeds $P_{\text {sts }}$. The power in equation (20) is therefore emitted as bremsstrahlung radiation if $P_{b} \geq P_{\text {sts }}$, which is equivalent to the condition

$$
\left(\Theta_{e}^{\prime}\right)^{1 / 2} \Theta_{e}^{2} \geq 0.1 r^{-1}
$$

which is usually satisfied, and we have used equations (2), (3), and (20). In Figure 1 we show that the increase in temperature and electron-positron number density across a typical central-engine collisional shock results in an increase by several orders of magnitude in bremsstrahlung emission rate across shocks of moderate to high Mach numbers. The Rankine-Hugoniot relations are taken from I89, the density of pairs in both the preshock and postshock regions is taken from Svensson $(1982,1984)$ and Bjornsson \& Svensson (1991), and the typical parameters of the twotemperature plasma are taken from WL89 at a distance of $r \sim 20$. This figure is taken from a more general discussion in CST96. Figure 1 gives the lower limit on the postshock radiation, because additional increase in emission due to, 


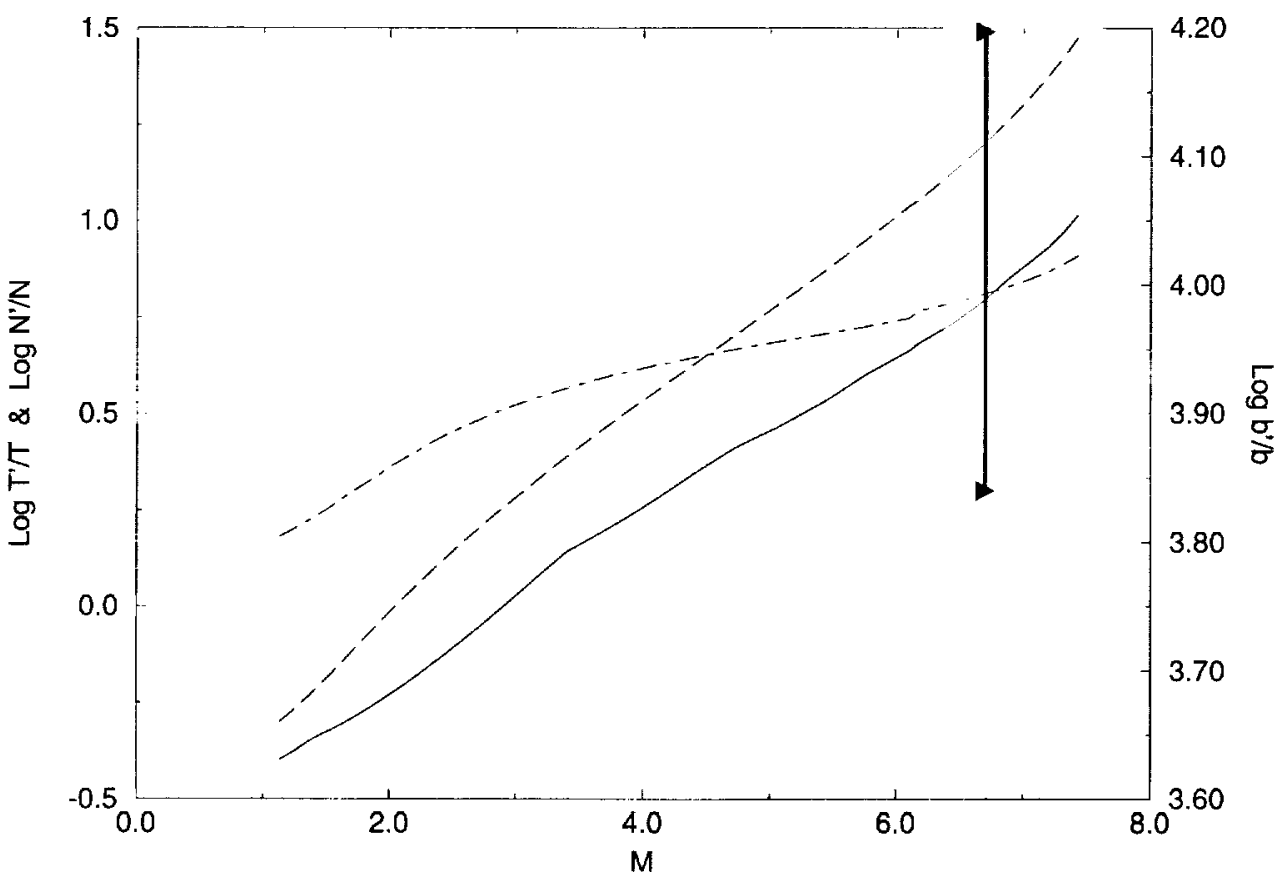

FIG. 1.-The effects of the increase in temperature, number density, and number of pairs across a typical central engine collisional shock with Mach number $M$ results in an increase in bremsstrahlung emission rate. The postshock-to-preshock ion temperature, ion density, and bremsstrahlung emissivity ratios, $T^{\prime} / T, N^{\prime} / N$, and $b^{\prime} / b$, are the dashed, dash-dotted, and solid curves, respectively. They are plotted vs. the Mach number. To the right of the thick solid line is the pair runaway region where condition (25) is fully satisfied. The pair rate equations the preshock and postshock regions in our code $i$ are taken from Svensson $(1982,1984)$ and Bjornsson \& Svensson (1991). The Rankine-Hugoniot relations for relativistic collisional plasmas are taken from I89. This figure is taken from a more general discussion in CST96. The preshock temperatures are taken from WL89 for $\dot{m} \sim 0.1$ at $r \sim 20$. The postshock ion temperature/ electron temperature ratio of roughly $\sim 10$, is appropriate for $\log N^{\prime} / N \sim 0.6$.

for example, Comptonization of the bremsstrahlung radiation, for example, is not taken into account. We therefore conclude that the postshock region can easily radiate away most of the power transferred from the star to the shock. We use this result in $\S 4$ for deriving a possible light curve from an emitting shock.

It has been suggested that for certain ranges of compactness the hot gas in the central engine is unstable, and the perturbations may result in the formation of a "twophase" region consisting of a dense cool (not completely ionized) phase and a hot phase (GR88). In a similar manner the postshock matter in our model may also form a twophase configuration. The postshock compactness is of order

$$
\begin{aligned}
\frac{\epsilon_{i}^{\prime}}{m_{e} c^{2} n_{i}^{\prime}} & \sim \frac{P_{\text {sts }}}{\lambda 2 \pi D_{*} c_{s} n_{i}^{\prime} m_{e} c^{2}} \\
& \sim 1600 \frac{\dot{m}}{r^{5 / 2} \sqrt{\Gamma \delta}} \Theta_{e}^{-2}\left(\frac{D_{*}}{R_{g}}\right)\left(\frac{\Omega}{4 \pi}\right)^{-1}\left(\frac{v_{\mathrm{acc}}}{v_{\mathrm{ff}}}\right)^{-1},
\end{aligned}
$$

where we have used equations (3) and (20) and assumed $\delta \sim 0.01$. This compactness is related to the standard compactness, $l=L \sigma_{\mathrm{T}} /\left(R m_{\mathrm{e}} c^{\mathbf{3}}\right)$ (Guilbert, Fabian, \& Rees 1983). As in GR88, the high compactness leads to an interesting effect: In some postshock regions ions are trapped and may lose most of their energy to the surrounding electronpositron pairs through a runaway cooling, which involves Comptonized bremsstrahlung radiation and then boundfree transitions. Counter to our experience with other shocks some postshock ions after being initially heated may cool below the original preshock temperature to a typical
GR88 cold phase temperature of

$$
T_{\mathrm{ps}} \geq\left[(1-A) \frac{L}{16 \sigma \pi R^{2}}\right]^{1 / 4},
$$

where $T_{\mathrm{ps}}$ is the postshock temperature and $A$ is the flux averaged albedo. This matter, which probably forms clouds of denser cold matter, may be "warm," i.e., partially ionized, because of its close proximity to the strong radiation from the nearby shock, or "cold" if the lifetime of the cold matter in the cloud is longer than the Keplerian timescale and the matter is not intensely irradiated.

A configuration with cold plasma in the central engine may therefore exist. The cold component may give rise to some emission and absorption features such as the gravitationally redshifted $\mathrm{Fe} \mathrm{K}$ line recently observed by $A S C A$ (Tanaka et al. 1995). The cold (or warm) phase may also eclipse the hot plasma under certain conditions. The eclipse may modify the primary emission by absorption in a postshock foamy sheet behind the Mach surface if $P_{\text {sts }}$ is expended on the cooling of the postshock matter, and if the lifetime of the cold blobs is long enough (see Appendix). In subsequent papers (CST96; STC96) we will present a full numerical solution for the rate equations in the two-phase postshock region, which accounts for these effects.

\subsubsection{Collisionless Shocks}

A naive application of the same procedure used in $\S 3.3 .1$ to collisionless shocks would have us compare the shock crossing time, which is either $t_{\mathrm{sw}} \sim r_{L i} / c_{\mathrm{A}}$ or the possibly longer $t_{\mathrm{sw}} \sim\left(r_{L i} / c_{\mathrm{A}}\right)\left(1+\omega_{\mathrm{b}} r_{\mathrm{Li}} / c_{\mathrm{A}}\right)$, which is increased due to confinement (where $\omega_{b}=e B / m c$ is the cyclotron frequency), 
to the deflection time $\lambda /(2 K T / m)^{1 / 2}$ (which is the typical timescale for, e.g., conduction losses). Shkarofsky, Johnston, \& Bachynski $\left(1966\right.$, p. 23) used these timescales $t_{\mathrm{sw}}$ for the diffusion time out from a confined region. The problem with this approach is twofold: (1) The width of a collisionless shock tends to be at least several Larmor radii (e.g., KEH85) and the crossing time is therefore several times $t_{B}$. (2) As discussed in $\S 2.2$ the plasma losses across collisionless shocks are determined not by single binary collisions, but rather by collective interaction between particles and self-consistently generated electric and magnetic fields. Most of the losses in collisionless shocks, (especially in the near virial fields limit), may be due to, for example, various microinstabilities in the collisionless plasma rather than conduction (Winske 1985; P85).

At present there are no analytical Rankine-Hugoniot relations for relativistic collisionless shocks in matter with density in excess of $n \sim 10^{10} \mathrm{~cm}^{-3}$. We therefore parameterize the increase in magnetic field magnitude, and possibly particle density and velocity, across the shock. This increase would result in an increase of the synchrotron and synchrotron-self-Compton radiation and would take place over a timescale similar to $t_{B}$.

We speculate that the Rankine-Hugoniot relations for the conditions envisioned in the central engine have some common features with the Rankine-Hugoniot relations of lower energy collisionless shocks (e.g., P85, and references therein). For simplicity's sake we assume that the quiescent radiation is due to synchrotron radiation only, such that

$$
L(0) \sim 2 \times 10^{-9} B^{2} \gamma n V_{\mathrm{X}},
$$

as in $\mathrm{B} 90$, where $L(0)$ is the actual observed bolometric luminosity and $V_{\mathrm{x}}$ is the volume of nonthermal emitting medium, which can be either in a quasi-spherical form or in a corona-over-a-disk form (see $\S 2$ ). The resulting postshock radiation density is therefore of order

$$
P_{s} \sim\left(\frac{n^{\prime}}{n}\right)\left(\frac{\gamma^{\prime}}{\gamma}\right)\left(\frac{B^{\prime}}{B}\right)^{2} \frac{L(0)}{V_{\mathbf{X}}} .
$$

We hence estimate the overall compactness of the postshock matter for quasi-spherical accretion:

$$
\frac{P_{s} \sigma_{\mathrm{T}} R_{\mathrm{X}}^{2}}{m_{e} c^{3}}=10 M_{6}^{-1}\left(\frac{\dot{m}}{0.01}\right)\left(\frac{r}{20}\right)^{-1}\left(\frac{n^{\prime}}{n}\right)\left(\frac{\gamma^{\prime}}{\gamma}\right)\left(\frac{B^{\prime}}{B}\right)^{2},
$$

and for coronal accretion:

$$
\frac{P_{s} \sigma_{\mathrm{T}} R_{\mathrm{X}} h}{m_{e} c^{3}}=10 M_{6}^{-1}\left(\frac{\dot{m}}{0.01}\right)\left(\frac{h}{20 R_{g}}\right)\left(\frac{r}{20}\right)^{-1}\left(\frac{n^{\prime}}{n}\right)\left(\frac{\gamma^{\prime}}{\gamma}\right)\left(\frac{B^{\prime}}{B}\right)^{2} .
$$

Extrapolating from interplanetary collisionless shocks (KEH85) and extragalactic collisionless shocks in jets (see Eilek \& Hughes 1991, and references therein) we speculate that the postshock magnetic field $B^{\prime}=\kappa B$ and density $n^{\prime}$ are larger than the corresponding preshock parameters. The compactness may therefore be increased enough for pair production to be important. The resultant pair production will be nonthermal, and the steepening of the spectrum of emission from the postshock region may be significant. This postshock emission will result in steepening of the overall spectrum if the postshock emission is comparable to that of the quiescent emission. Given a large perturbation and a strong shock with high $\mathscr{M}_{\mathrm{A}}$ number (see $§ 2.2$ ) the postshock parameters may result in a significant increase of the flux and the steepening of the spectrum. We discuss the implication of this result in STC96.

\section{MODIFICATION OF THE CONTINUUM SOURCE}

\subsection{Effects of Radiative Shocks}

Since the speed of the "star" in our model is assumed to be roughly $\sim 0.2 c$ at $r=20$ we will neglect the increase in shock radiation due to the Doppler effect (for corrections see STC96). We note, however, that the angle spanned by the shock front area vector and the line of sight determines the percentage of outcoming shock radiation due to the postshock eclipsing, which is dealt with in the next subsection. Therefore, emission only doubles when the shock front is directed toward the observer (see Fig. 2). In our approximation we assume that the spectral index in the postshock pair-dominated region is $\sim 1$, as was shown by DF89, CB91, TK95, and others, whereas in the ambient matter the spectral index is $\sim 0.5$, as is expected in synchrotron and synchrotron-self-Compton sources (B90, and references therein).

In Figure 3 we show the effect of a shock on the spectral index in the soft X-ray waveband in the $0.2-2 \mathrm{keV}$ window, the hard X-ray waveband in the $10.0-20.0 \mathrm{keV}$ window, and the very hard $100.0-300.0 \mathrm{keV}$ window. The angle $\beta$ is spanned by the shocking star, the central engine and the observer-black hole line of sight. One can clearly see that the outcoming flare is soft as observed in several compact Seyfert galaxies. The lesser softening in the higher energy bands is also consistent with observations (see MDP93). We assumed that the maximum observed flux with spectral index of order unity in the $0.1-300 \mathrm{keV}$ range is similar to the flux from the ambient matter. We note that a spectral break near $300 \mathrm{keV}$ was assumed in our work because we assumed a two-dimensional slab corona configuration for our pair spectrum, as in TK95, which results in a spectral break near $\sim 300 \mathrm{keV}$ for high enough compactness. A combination of observations with present and future missions, such as $X T E, X M M$, and $A X A F$, may be able to determine if the light curves fit these characteristics.

\subsection{Effects of Postshock Cold Matter}

Here we obtain the light curve for radiation from hot plasmas in the central engine, which is "eclipsed" by the cold postshock matter. The central engine will be eclipsed when the postshock cold matter creation timescale, of order $t_{e}$ in equation (23), is shorter than the cold matter timescale $t_{s}$ (eq. [A9] in the Appendix), and when the postshock matter column density is of order $n^{\prime} c_{s} t_{s} \sim 10^{21-24} \mathrm{~cm}^{-2}$ (depending on the ionization state of the postshock "cold" matter). Both conditions are met for postshock matter in at least some AGNs (see eqs. [2], [3], [7], [24], and [A9]), and an eclipse of the central engine is therefore possible.

In obtaining the light curve our approach is geometrical in nature. We assume that a conical shock front, similar in shape to a simple Mach cone (see $\S 2$ ) eclipses a circular continuum source. To highlight the geometrical effects we also chose a rectangular continuum source. A possible picture of what happens near the central region of an AGN with an inhomogeneity is shown in Figure 2. Here a shock inducing star (at the tip of the cone) orbits inside a thick accretion torus, which is located outside the X-ray contin- 


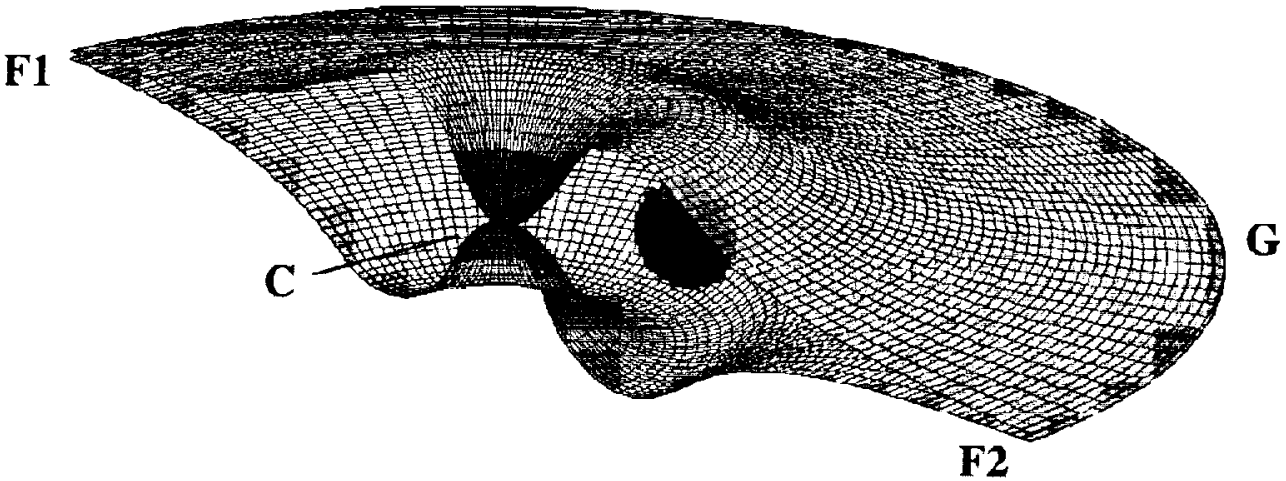

FIG. 2.-Doughnut-shaped ion torus shown here (not a linear scale) consists of an inner part of a thin disk (at points F1 and F2), the central black hole (at point $C$ ) and the continuum $X$-ray source (shaded in gray, near the black hole). The rest of the ion torus is filled with optically thin two-temperature plasma. the "Mach-cone" optically and shocks the two-temperature plasma. When the shock front moves toward the observer a flare is seen. Behind the surface of observer is in the direction of point $G$.

uum region but confined to a region smaller than the thin outer disk (points $F 1$ and $F 2$ are the inner edges of the thin disk). From an observer's point of view the points $O, P$, and $Q$ on the Mach cone (Fig. 4) form a "fan" in the shape of a triangle that partially obscures the internal continuum source. The type of occultation depends on the optical depth of the matter on and near the shocked surface.

Since the extent of the optically thick shock may be limited, as was shown in $\S 3$, we choose the point of view of

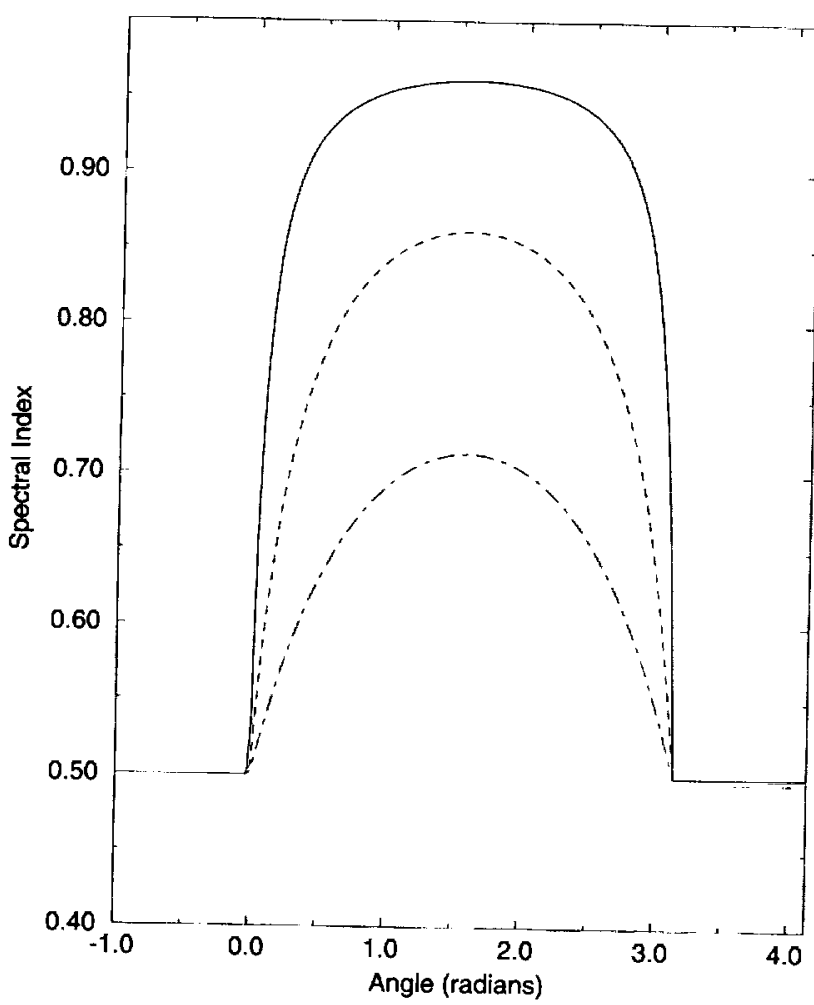

Fig. 3.-Effect of a shock on the spectral index of flux from the central engine with a radiative shock front is plotted vs. the angle spanned by the Keplerian perturbation, the central black hole and the line of sight. The solid line is for flux in the "soft" $0.2-2 \mathrm{keV}$ window, the dashed line is for flux in the "hard" 10-20 keV window, and the dash-dotted line is for the $100-300 \mathrm{keV}$ window. The trend of harder flares in the higher for the bands is apparent. At an angle $\pi / 2$ the Mach cone is pointing toward the observer. an observer in direction $G$, which requires the shortest extent of optically thick matter. Figure 4 is a simplification of the observer's view, which is used for the calculation of the light curve. It is based on the following assumptions:

1. The continuum $X$-ray emitting region is assumed to be circular, with homogeneous surface brightness, as viewed when unocculted. Its radius is $R=R_{\mathrm{X}}$. We note that in an alternative model, in which the soft X-ray region is larger than the hard $X$-ray region, the soft minimum flux will be significantly delayed with respect to the hard flux.

2. The Mach surface is assumed to be an equilateral triangle, passing across the center of the circular source.

3. Density variations are simplified by assuming three density regions. In region 1 (angles smaller than $\phi_{1}$ ) the matter is opaque to both hard and soft X-rays. In region 2, with angles between $\phi_{1}$ and $\phi$, the matter is transparent to hard X-rays, but opaque to soft X-rays. In region 3 , behind the darker triangle, the density of matter varies with distance, due to the spread of matter, and is partially transparent. We consider only the hard X-ray waveband in the $10-20 \mathrm{keV}$ window in which no absorption is expected and the soft $\mathrm{X}$-ray waveband in the $0.5-2 \mathrm{keV}$ window in which

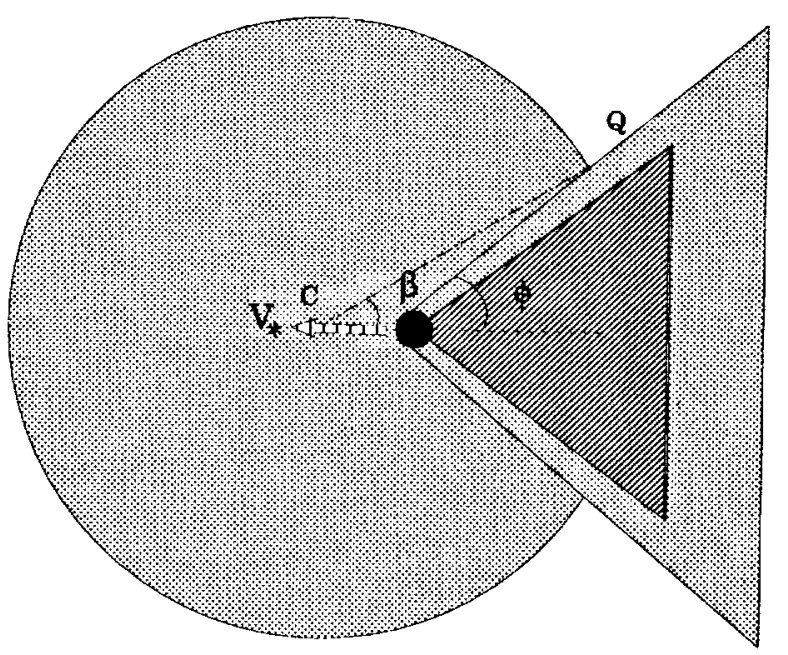

FIG. 4.-Observer's view of the AGN in our model with covering factor of $f=0.5$. This is a simplified form of the view from point $\mathrm{G}$ in Fig. 2 . The stepwise fashion, which was chosen to describe the absorber, is for demonstrative purposes. See $\$ 4$ for details. 


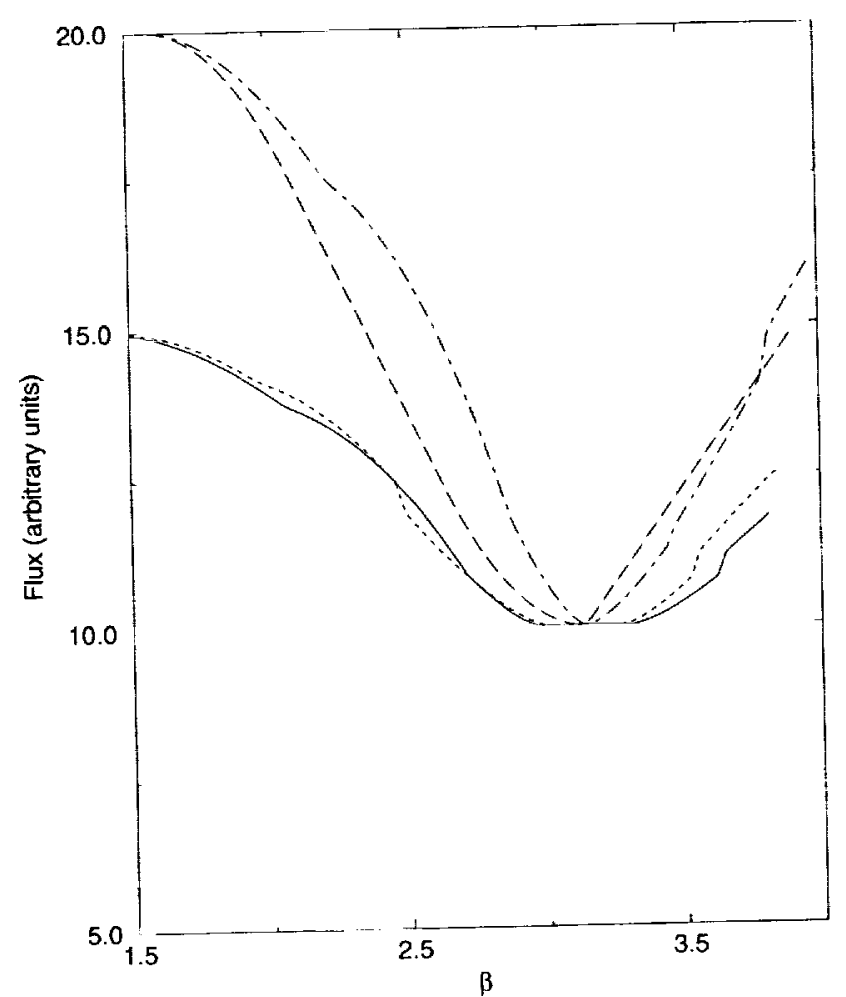

Fig. 5.- Light curves near a dip that will be observed when the source is eclipsed by the postshock matter is shown for the softer (dash-dotted curve) and the harder (solid curve) X-ray windows with $\phi=45^{\circ}$. The short curve) and the harder (solid curve) X-raller Mach surface opening angle of dashed curve is for the case of a smaller Mach surface opening angle of $\phi=30^{\circ}$, for the hard X-rays. The long-dashed curve is for the simplified $\phi=3$ in which a triangle with an opening angle $\phi=45^{\circ}$ eclipses a square source. In all cases the maximum covering factor is $f=0.5$ (see $\S 4$ for details).

absorption due to cold or warm oxygen is expected. We chose the maximum covering factor to be $f=0.5$ for demonstrative purposes.

4. The strength of the predip flare was assumed to be negligible for demonstrative purposes.

In Figure 5 we see the light curves near a dip in two energy bands that will be observed when the source is eclipsed by the postshock matter. With a Mach surface opening angle of $\phi=45^{\circ}$, the softer X-ray light curve is represented by the dash-dotted line and the harder X-ray light curve by the solid line. The dotted curve is for the case of a smaller opening angle of $\phi=30^{\circ}$, for the hard X-rays. The dashed curve is for the simplified case in which a triangle with an opening angle $\phi=45^{\circ}$ eclipses a square source. In all cases the maximum covering factor is $f=0.5$.

The decrease of flux toward the dip is energy dependent. A delay is observed in the sense that the decrease in photon count in higher energies lags the decrease in the lower energies. The light curve in the hardest window has a cusplike shape, dropping to half its value at the bottom. The bottom was chosen to be at half the intensity so that the size of the central sector of absorbing material within $\phi_{1}$, which is opaque to the harder $\mathrm{X}$-rays, is approximately half the size of the harder $\mathrm{X}$-ray source size during maximal occultation. The maximum value for $R_{\mathrm{X}}$ is thus obtained with $\phi_{1}=45^{\circ}$.

\section{DISCUSSION}

The results of $\S \S 2,3$, and 4 imply that given a perturbation, an accretion rate and a black hole mass one could calculate temporal features and confirm the "condensation" of cold (not completely ionized) matter. Our work thus complements the work by GR 88 and LW88. We also argue that one could also calculate the variability and power spectrum, the rate of angular momentum transfer, and the effects of particle acceleration. The results of $\S \S 2$ and 3 may give rise to the prediction of the $r_{h s}=l_{h} / l_{s}$ ratio, $l_{h}$ and $l_{s}$, where $l_{h}$ is the compactness of the hard nonthermal radiation and $l_{s}$ is the compactness of the soft thermal "seed photons" (see STC96).

When the accretion rate is relatively low one expects a disk corona configuration. In this configuration accretion disk instabilities are expected to yield microvariability (e.g., W93). As argued in $\$ \$ 2$ and 3 collisionless shocks will then tend to spread these perturbations throughout the corona at the fastest magnetosonic speed, thus enabling large amplitude variability. Since the coronal compactness is relatively small only a fraction of the perturbation energy will be radiated away (and most of the perturbation may travel outward as winds). Note, however, that various microinstabilities may increase the efficiency of energy transfer.

The radiation will tend to be relatively hard since no Comptonized bremsstrahlung radiation is expected and only synchrotron-self-Compton (SSC) or nonthermal pairs cascades are possible locally, with a very hard outcoming spectrum (e.g., B90; TK95). The compactness $l_{s}$ also depends on $\dot{m}$ (FKR92), and $r_{h s}$ may not change as fast as $l_{h}$. The radiation may be SSC only if $l_{h}+l_{s}$ is such that small IR blocking cold cloudlets form in the shocked material (as in CFR92), or else IR-X-rays correlation should be observable (Done et al. 1991). Reflection of the hard radiation from the relatively cold disk component may result in an $\mathrm{Fe}$ emission line, such as the one recently observed by $A S C A$ (Tanaka et al. 1995, and references therein).

When the accretion rate is relatively high $(\dot{m} \geq 1)$ one may expect a very dense corona or a thick two-temperature torus. Perturbations will be the result of instabilities in the disk (e.g., Narayan 1990, and references therein) and the resultant shocks will produce bremsstrahlung-Compton radiation or may even result in a two-component Guilbert $\&$ Rees medium. The emission from such media becomes softer when $l_{h}+l_{s}$ become higher (ST93; Nandra \& George 1994). In general one expects $l_{s}$ to increase more than $l_{h}$ in this case, because of the soft photons that originate or are reprocessed in the cold component.

The emission spectrum for the high $\dot{m}$ is dominated by a more thermal-like component with a possible hightemperature bremsstrahlung, which may imply AGN evolution, which is thought to be responsible for the observed X-ray background.

Shocks can naturally explain a common feature of the spectra of most AGNs. In the spectra of radio-quiet AGNs the ratio of thermal to nonthermal emission is probably of the order $0.3 \leq L_{T} / L_{N T} \leq 3$, where $L_{T}$ is the thermal luminosity and $L_{N T}$ is the power-law nonthermal luminosity (LW88). This result is readily explained if the hot continuum emitting plasma is nearly optically thick (Sivron 1995). A mechanism in which the optical depth is limited to unity involves a two-phase medium: A medium that would have been optically thick if the accretion were spherical becomes a two-phase medium with decreased optical depth for the hot phase whenever the optical thickness exceeds unity, or equivalently whenever the luminosity is near-Eddington 
(ST93; STC96; GR88). Such two-phase media are naturally produced with shocks, because shocks are so readily available in the central engine (as was shown in $\S 2$ ) and because shocks naturally channel the energy of perturbations into radiative cooling, which cools portions of the hot phase in the case of near-Eddington luminosities (as was shown in $\S$ 3). The cycle is completed when the speed of sound in the rarefied hot phase again falls below the Keplerian velocity due to the "evaporation" of cold phase clouds, which is usually faster than the dynamical timescales, or due to cooling processes in the hot plasma, and shocks are again possible (for a typical timescale of "evaporation" see, e.g., Zel'dovich \& Raizer 1967, p. 571 and the Appendix).

If radio-quiet AGN central engines have a disk corona geometry, the observed spectra cannot be explained by homogeneous coronas (Fabian et al. 1995; Stern et al. 1995; Iwasawa et al. 1996). This paper establishes the presence of shocks that naturally disrupt the homogeneous corona. Perturbations in the hot homogeneous coronas may be considered in the sense described in this paper in order to establish the true geometrical shape of the inhomogeneous corona and calculate the resultant spectrum.

Shocks may also explain the observed power spectrum of the central engine. A weakness of our assumption that a single star is responsible for the shock and resultant emission is that periodicity, which has not been observed in AGNs, may be expected. A star orbiting a supermassive black hole can be a curiosity, or a rule-depending on future observations, but from $\S 3.2$ it is apparent that any large perturbation present in the innermost $20 R_{g}$ can yield the luminosity change of equation (20). It is therefore probably more likely that the power spectrum is the result of perturbations that are not associated with a captured star. If the conditions in equations (4) and (30) are met for these perturbations, and if perturbations of characteristic size, time, and brightness occur at a random distance from the black hole, the resultant power spectrum slope will be between that of random shot noise and chaotic noise. Our subsequent work (STC96) uses the arguments of Sunyaev \& Titarchuk (1980) and DF89 to obtain these power spectra, which are comparable to the power spectra observed in radio-quiet AGNs (Green 1993, and references therein). The condition in equation (30) on the compactness then determines that a one-dimensional introduction of perturbations, rather than the two-dimensional introduction of perturbations as in thin disk models, is needed in order to reproduce the observed power spectra. The physics of random introduction of perturbations in one dimension may be easier to explain than the random introduction of perturbations in two dimensions (STC96).Condition (29), or an equivalent for a nonspherical perturbation, may result in a high-frequency cutoff in the power spectrum (STC96).

If the shocks are collisionless the source may not be optically thick, and modification through pair creation may not be as significant, unless the fields are near virial. In the case of collisionless shocks that do not satisfy the conditions in equations (32)-(34), one should note that energy may still be deposited by the shock to the ambient hot plasma. Various microinstabilities may still result in microvariability, but large-amplitude variability is harder to produce.

In the above discussion of variability we did not try to calculate the timescale in which the postshock matter returns to its original state. We generally assumed that the recovery timescale is shorter than the dynamical timescales.
If the recovery timescale is larger than the dynamical timescale, the power spectrum is further modified. The effect of a two-phase medium on the variability is also calculated in our subsequent work (STC96).

The spectral variability of flares and dip described in $\S \S 3$ and 4 was already found to be similar to that of several Seyfert 1 galaxies such as NGC 4051 (Kunieda et al. 1990), MCG -6-30-15 (Nandra, Pounds, \& Stewart 1990; Matsuoka et al. 1990; Fabian et al. 1994; Otani et al. 1995; Sivron et al. 1996a), and others (MDP93). The similarity is not only in the sense that the power-law spectral slope is anticorrelated with the overall flux, but also in the sense that the low flux level is correlated with some emission and absorption features. Although this correlation was traditionally explained to be the result of matter in the line of sight, which is far from the central engine (e.g., Nandra et al. 1990 ) it is generally hard to explain why this matter is always smaller in thickness than the size of the central engine (Sivron et al. 1996a). The emission and absorption features may thus be associated with an increase in optical depth of cold matter within, or very close to, the central engine, as in the case described in $\$ 4.2$.

Shocks and their by-products may be used for the outward transfer of angular momentum in the central engine (STC96). Spiral shocks, which were considered for the outward transfer of angular momentum in the outer disk, may form when a massive companion perturbs the disk (Savonije, Papaloizou, \& Lin 1994, and references therein, in reference to galactic sources). Angular momentum can be transferred in this way in AGN inner disks as well (Chakrabarti 1995). Spiral shocks probably take a completely different geometrical shape in compact central engines, where the geometry of the emitting material is not that of a thin disk. The angular momentum transfer in the central engine in high-accretion Seyfert 1 galaxies and radio-quiet quasars can be due to chaotic, rather than spiral, shock structure (STC96).

In the case of lower accretion rates significant outflows must also be considered as a part of the total angular momentum transfer, since the optical depth is limited and mass ejection may become significant. However, the availability of shocks in the central engine and the presence of mechanisms that can disturb the axisymmetrical structures makes it imperative that shock-related angular momentum transfer is significant. Examples of mechanisms that can disturb the axisymmetry include a poloidal magnetic field structure, a Lense-Thirring precession of tilted axisymmetrical structures in a Kerr geometry, etc.

Shocks may also be an indirect agent in the outward transfer of angular momentum. The postshock cold clouds may transfer angular momentum through binary and triple collisions (STC96). Because the hot component in a GR88 two-phase model is fairly optically thin, some cold clouds may also be ejected carrying with them large amounts of angular momentum.

Other processes affected by the presence of shocks include particle acceleration and pair production. Many models use an input energy spectrum for a large nonthermal electron population in order to obtain their model spectra (B90, and references therein; CB90). From our shock model one should directly calculate the normalization of this distribution by utilizing, for example, the theory of Fermi and shock-Fermi acceleration for a specific postshock preshock compression ratio (Blandford \& Eichler 1987; Levinson 
1994). We note that modifications due to Fermi acceleration will probably result in the lowering of cooling efficiency, as in equation (30). That a significant portion of the particles may gain a nonthermal high-energy distribution function was anticipated (but not calculated) by many previous authors that discussed pair modifications of spectrum and structure of the central engine (see Guilbert et al. 1983; Begelman, Sikora, \& Rees 1987; Tritz \& Tsuruta 1989; Kusunose \& Takahara 1988; CB90; GH94).

\section{CONCLUSIONS}

We have shown that shocks in the central engines of AGNs form for a wide range of accretion parameters. We have also shown that perturbations in the central engine will effectively radiate their energy away through shocks and that for a wide range of accretion parameters that radiation will be observable. Given the generality of our results we believe that shocks should be fairly common and that their effects on observations should be significant.

We thank the anonymous referee for some very helpful remarks. We thank M. J. Rees, N. Iwamoto, and N. Sivron for useful discussions and comments. We also thank W. McHargue and M. J. Kellen for their help with supporting software. This work was supported in part by NASA grants NAGW-2208 and NAG8-230 and NSF grant RII-8921978.

\section{APPENDIX}

The lifetime of the thermal gas sheets and filaments that form behind the shock front can be evaluated using one of the two following methods:

The minimum lifetime of plasma sheets and filaments can easily be approximated for the case in which the "sheets" are in the shape of a spherical cloud and all of the incident radiation is transformed into an energy increase of size $\Delta E$ :

$$
t_{*} \geq \frac{\Delta E}{F}=140 r^{1 / 2}\left(\frac{r_{c}}{c}\right)\left(\frac{k T_{c}}{\mu m_{\mathrm{H}} c^{2}}\right)
$$

where $F$ is the maximum flux incident on a cloud, $r_{c}$ is the radius of a cloud and $T_{c} \sim 10^{8} \mathrm{~K}$ is the final temperature of the clouds in which all the elements are completely ionized (see also Bond \& Matsuoka 1993). For a typical cloud of size $10^{8} \mathrm{~cm}$ (for $M \sim 10 M_{6}$, using the same arguments as in CFR92), the lifetime of the clouds is greater than $10^{-4} \mathrm{~s}$. This result is a lower limit, because no radiative losses are considered in spite of the fact that bound-free and free-free emissions, which are the dominant mechanisms in the creation of such thermal matter, have already been proven to be efficiently radiating energy in § 3.2. Whereas this timescale describes the dissipation of a cloud, we next try to determine the beginning of such a process, which we show to have a longer timescale.

We assume that the temperature decrease is due to adiabatic expansion into vacuum and calculate the subsequent timescale necessary for the optical depth to be halved. The following equations govern the adiabatic expansion of clouds. The continuity equation in spherical coordinates, for a spherically symmetric time-dependent case (LL87), is

$$
\frac{\partial \rho}{\partial t}+\frac{1}{r^{2}} \frac{d}{d r}\left(r^{2} \rho v\right)=0,
$$

where $r$ is the radius of the exploding plasma cloud and $v$ is the velocity of a fluid element. The Euler equation is

$$
\frac{d v}{d t}+\frac{1}{\rho} \frac{d P}{d r}=0
$$

The adiabatic (energy) equation is

$$
P=T^{\gamma /(\gamma-1)},
$$

where $\gamma=5 / 3$ is roughly the adiabatic index for an ideal monoatomic gas, for hydrogen-dominated plasmas. The equation of state is

$$
T \sim \frac{m_{\mathrm{H}} P}{\rho k_{\mathrm{B}}}
$$

The total mass of a cloud, $M_{\mathrm{cld}}$, is

$$
M_{\mathrm{cld}}=\frac{4 \pi}{3} r^{3} \rho
$$

The optical depth of a cloud, assumed here to be bremsstrahlung and bound-free dominated, is

$$
\tau \sim 1.0 \times 10^{3} r T^{-1 / 2} Z^{2} \rho^{2}\left(\frac{h v}{\mathrm{keV}}\right)^{-3}
$$

The expansion of the clouds results in their transparency. The optical depth of the clouds as they expand (and are assumed to be opaque, $\tau=1$, at that size), can be related to the corresponding variations in cloud size, density, and temperature without the need to solve equations (A2) and (A3) explicitly. By substituting equation (A6) into equation (A5) and then into 
equation (A4), we get $T \propto r^{3-3 \gamma}$, and substituting the result into equation (A7) we find

$$
\frac{\tau\left(r_{0}\right)}{\tau\left(r_{f}\right)}=\left(\frac{r_{f}}{r_{0}}\right)^{6}
$$

Here $r_{f}$ is the final size of the expanding cloud. From this we can see that a bremsstrahlung and bound-free dominated, adiabatically expanding cloud will only need to grow by $r_{f} / r_{0} \sim 1.12$ for the optical depth to decrease by a factor of 2 . Substituting these initial and final sizes into equation (A3) we can estimate the lifetime of a cloud. Since the pressure, density, and size change very little when a cloud opacity is halved, the Euler equation becomes roughly $d^{2} r / d t^{2} \sim-\left(1 / \rho_{0}\right)\left(P_{0} / r_{0}\right)$. Integrating twice, using the initial conditions $v(t=0)=0$ and $r(t=0)=r_{0}$, we get

$$
t_{s}>\sim(100 \mathrm{~s}) \frac{\left(r_{8}\right)^{2}}{T_{6}},
$$

where $r_{8}=r_{0} / 10^{8} \mathrm{~cm}, T_{6}=T / 10^{6} \mathrm{~K}, r_{0}$ and $P_{0}$ are the initial size and pressure of the dense sheets, and $t_{s}$ is the time from the beginning of expansion. Hence, the postshock sheets become transparent on a timescale of hundreds of seconds. As a result a "curtain" of cold matter of thickness $t_{s} c_{s}$ is comoving with the Mach cone. This curtain may partially obscure the radiation from the central engine if its optical depth, $n^{\prime} t_{s} c_{s} \sigma$, where $n^{\prime}$ is the postshock cold matter average density and $\sigma$ is the cold matter's effective cross section, is nearly unity. Because of confining pressure from magnetic fields, which may appear on the right-hand side of equation (A3), the lifetime of a cloud may be longer than that in equation (A9).

Considering the velocity of the inhomogeneity found in $\S 2$ and the fact that the postshock matter moves at subsonic velocity, this result determines that the clouds form a "foamy" wall of thickness $\sim c_{s} t_{s} \sim 10^{10} \mathrm{~cm}$. A typical filling factor of $C \sim 0.01$ is reasonable for the ratio of ambient and thermal matter under the assumption that half the ambient matter becomes thermal in the postshock region (with the necessary covering factor $\sim 1$; see CFR92 and ST93). Therefore, on the average, one sheet of thickness $10^{8} \mathrm{~cm}$ can partially eclipse the continuum source.

\section{REFERENCES}

Abramowicz, M. A. 1990, in Structure and Properties of Accretion Disks, ed. C. Bertout, S. Collin, \& J. P. Lasota (Gif-sur-Yvette: Editions Frontiéres), 299

Abramowicz, M. A., Calvani, M., \& Nobili, L. 1980, ApJ, 242, 772

Artemova, I. V., Bisnovatyi-Kogan, G. S., Bjornsson, G., \& Novikov, I. 1996, MNRAS, in press

Barnes, A. 1985, ApJ, 265, 457

Begelman, M., Sikora, M., \& Rees, M. J. 1987, ApJ, 313, 643

Bjornsson, G. \& Svensson, R. 1991, MNRAS, 249, 177

Blandford, R. D. 1990, in Active Galactic Nuclei, Saas-Fee Advanced Course 20 , ed. T. J.-L. Courvoisier \& M. Mayor (Berlin: Springer), 161 (B90)

Blandford, R. D., \& Eichler, D. 1987, Phys. Rep., 154, 1

Blandford, R. D., \& Narayan, R. 1993, ARA\&A, 30, 311

Bond, I. A., \& Matsuoka, M. 1993, MNRAS, 265, 619

Caditz, D., Sivron, R., \& Tsuruta, S. 1996, in preparation (CST96

Celotti, A., Fabian, A. C., \& Rees, M. J. 1992, MNRAS, 255, 419 (CFR92)

Coppi, P., \& Blandford, R. D. 1990, MNRAS, 245, 453 (CB90)

Chakarbarti, S. K. 1995 , ApJ, 464, 664

Devries, M., \& Kuijpers, J. 1989, in Two-Topics in X-Ray Astronomy, ed. J. Hunt \& B. Battrick (ESALAB SP-296), 1069

Done, C., \& Fabian, A. C. 1989, MNRAS, 240, 81 (DF89)

Done, C., Ward, M. J., Fabian, A. C., Kunieda, H., Tsuruta, S., Lawrence, A. Smith, M. G. \& Wamsteker, W. 1991, MNRAS, 243, 713

Eilek, J. A., \& Hughes, P. A. 1991, in Beam and Jets in Astrophysics, ed. P. A. Hughes (Cambridge: Cambridge Univ. Press), 428

Fabian, A. C. \& the ASCA P. V. Team. 1994, PASJ, 46, L59

Fabian, A. C., Nandra, K., Reynolds, C. S., Brandt, W. N., Otani, C., Tanaka, Y., Inoue, H., \& Iwasawa, K. 1995, MNRAS, 277, L11

Frank, J., King, A. R., \& Raine, D. J. 1992, Accretion Power in Astrophysics (Cambridge: Cambridge Univ. Press) (FKR92)

Ghisellini, G., \& Haardt, F. 1994, ApJ, 429, L53 (GH94)

Green, A. 1993, Ph.D. thesis, Univ. Southampton, UK

Guilbert, P. W., Fabian, A. C., \& Rees, M. J. 1983, MNRAS, 205, 593

Guilbert, P. W., \& Rees, M. J. 1988, MNRAS, 233, 475 (GR88)

Haardt, F., \& Maraschi, L. 1991, ApJ, 380, L51

Iwamoto, N. 1989, Phys. Rev. A, 39, 4076 (189)

Iwasawa, K., Fabian, A. C., Mushotzky, R. F., Brandt, W. N., Awaki, H., \& Kunieda, H. 1996, MNRAS, in press

Kennel, C. F., Edmiston, J. P., \& Hada, T. 1985, Geophys. Monographs, 34, 1 (KEH85)

Kunieda, H., Hayakawa, S., Tawara, Y., Koyama, K., Tsuruta, S., \& Leighly, K. 1990, ApJ, 384, 482

Kusunose, M., \& Takahara, F. 1988, PASJ, 40, 435

Landau, L. D., \& Lifshitz, E. M. 1987, Fluid Mechanics (2d ed; Oxford: Pergamon) (LL87)

Levinson, A. 1994, ApJ, 426, 327

Liang, E. P. T., \& Price, H. 1977, ApJ, 218, 247

Lightman, A. P., \& White, T. 1988, ApJ, 335, 57 (LW88)

Lightman, A. P., \& Zdziarski, A. A. 1987, ApJ, 319,643

Matsuoka, M., Piro, L., Yamauchi, M., \& Murakami, T. 1990, ApJ, 361, 440
McHardy, I. 1988, Mem. Soc. Astron. Italiana, 59, 239

Mushotzky, R., Done, C., \& Pounds, K. 1993, ARA\&A, 31, 717 (MDP93)

Nandra, K., \& George, I. M. 1994, MNRAS, 267, 974

Nandra, K., Pounds, K. A., \& Stewart, G. C. 1990, MNRAS, 242, 660

Narayan, R. 1990, in Structure and Properties of Accretion Disks, ed. C. Bertout, S. Collin, \& J. P. Lasota (Gif-sur-Yvette: Editions Frontieres), 153

Narayan, R., \& Yi, I. 1994, ApJ, 428, L13

Otani, C., et al. 1996, PASJ, in press

Papadopoulos, K. 1985, Geophys. Monographs, 34, 59 (P85)

Papaloizou, J. C. B., \& Pringle, J. E. 1984, MNRAS, 208, 721

Pineault, S., \& Landry, S. 1994, MNRAS, 267, 557

Pudritz R. E. \& Fahlman, G. G. 1982, MNRAS, 198, 689

Qian, S. J., Quirrenbach, A., Witzel, A., Krichbaum, T. P., Hummel, C. A., \& Zensus, J. A. 1991, A\&A, 241, 15

Rees, M. J. 1984, ARA\&A, 22, 471

Rees, M. J., Begelman, M. C., Blandford, R. D., \& Phinney, E. S. 1982, Nature, 295,7

Savonije, G. J., Papaloizou, J. C. B., \& Lin, D. N. C. 1994, MNRAS, 268, 13

Shapiro, S. L., Lightman, A. P., \& Eardley, D. M. 1976, ApJ, 203, 187

Shkarofski, I. P., Johnston, T. W., \& Bachynski, M. P. 1966, The Particle Kinetics of Plasmas (Reading: Addison-Wesley)

Sivron, R. 1995, Ph.D. thesis, Montana State Univ.

Sivron, R., Kunieda, H., Tsusaka, Y., \& Tsuruta, S. 1996a, in preparation

Sivron, R., \& Tsuruta, S. 1993, ApJ, 402, 420 (ST93)

. 1994, BAAS, 26, 183

Sivron, R., Tsuruta, S., \& Caditz, D. 1996b, in preparation (STC96)

Stern, B. E., Poutanene, J., Svennson, R., Sikora, M., \& Begelman, M. C. $1995, \mathrm{ApJ}, 449, \mathrm{~L} 13$

Sunyaev, R. A., \& Titarchuk, L. G. 1980, A\&A, 86, 121

Svensson, R. 1982, ApJ, 258, 321

.1984, MNRAS, 209, 175

Svensson, R., \& Zdziarski, A. A. 1994, ApJ, 436, 599

Syer, D., Clarke, C. J., \& Rees, M. J. 1990, MNRAS, 250, 505 (SCR90)

Tanaka, Y. et al. 1995, Nature, 375,659

Taub, A. H. 1948, Phys. Rev., 74, 328

Thorne, K. S., \& Price, R. 1975, ApJ, 195, L101

Tritz, B., \& Tsuruta, S. 1989, ApJ, 340, 203

Tsuruta, S., \& Kellen, M. J. 1995, ApJ, 453, L9 (TK95)

White, T. R., \& Lightman, A. P. 1989, ApJ, 340, 1024 (WL89)

Wiita, P. J. 1993, Proc. 6th Guo Shou Jing Workshop: Accretion and Jets in Astrophysics, ed. L. Qibin, Y. Lantian, X. Guangshong, \& Y. Pibo, (Wuhan: Huazhong Nurmal Univ. Press), 1 (W93)

Winske, D. 1985, Geophys. Monographs 34, 225

Zel'dovich, Y. B., \& Raizer, Y. P. 1966, in Physics of Shock Waves and High-Temperature Hydrodynamic Phenomena (New York: Academic Press)

Życki, P. T., Collin-Souffrin, S., \& Czerny, B. 1995, MNRAS, 277, 70 (ZCC95) 
